\title{
Evaluating condom catheter balloon tamponade in non-traumatic postpartum haemorrhage resistant to medical management
}

\author{
Syed Nawaz Ahmad*, Sunita Seth, Shefali Agarwal, Swati Choudhary
}

Department of Obstetrics and Gynecology, Deen Dayal Upadhyay Hospital, New Delhi, India

Received: 26 March 2016

Revised: 01 May 2016

Accepted: 13 May 2016

\section{*Correspondence:}

Dr. Syed Nawaz Ahmad,

E-mail: dr.syednawaz@gmail.com

Copyright: (c) the author(s), publisher and licensee Medip Academy. This is an open-access article distributed under the terms of the Creative Commons Attribution Non-Commercial License, which permits unrestricted non-commercial use, distribution, and reproduction in any medium, provided the original work is properly cited.

\begin{abstract}
Background: Obstetric haemorrhage remains the most important cause of maternal mortality worldwide accounting for $25 \%$ of maternal deaths annually. The aim of the study was to evaluate efficacy of a condom catheter assembly for uterine tamponade in the management of non-traumatic postpartum haemorrhage (PPH).

Methods: It was a prospective interventional study done in a tertiary care hospital in New Delhi, India. Thirty three women with intractable PPH unresponsive to medical management were managed by uterine balloon tamponade using a condom-catheter assembly prior to surgical intervention.

Results: The catheter successfully controlled haemorrhage in 31 out of 33 patients. In both the failed cases, hysterectomy was required. Among the failed cases there was one maternal death due to sepsis and multi-organ dysfunction syndrome (MODS). In cases where the balloon was successful, it was removed around 24 hours later and no further bleeding or complication was observed.

Conclusions: Placement of a condom catheter balloon can successfully treat non-traumatic PPH refractory to medical management. It is simple, inexpensive, easily, available and in those with successful placement no procedure related morbidity was observed. The potential for it to be used by inexperienced operators in areas with limited resources makes it a useful tool in management of PPH.
\end{abstract}

Keywords: Postpartum haemorrhage, Condom catheter balloon, Non-traumatic

\section{INTRODUCTION}

Obstetric haemorrhage remains the most important cause of maternal mortality worldwide accounting for $25 \%$ of maternal deaths annually. It is estimated that worldwide one woman dies every four minutes due to postpartum haemorrhage with an average yearly incidence of about $1,40,000$ women deaths. ${ }^{1}$ Of the numerous causes of postpartum haemorrhage, uterine atony is the commonest, complicating 1 in 20 pregnancies. However, death from PPH can largely be avoided through proper prevention, diagnosis and management. ${ }^{2}$ Doumouchtsis SK et al in a systematic review to identify all the studies evaluated the success rates of treatment of PPH with uterine balloon tamponade, uterine compression sutures, pelvic devascularisation and arterial embolization. ${ }^{3}$ The cumulative outcomes showed success rates of $84 \%(95 \%$ CI, $77.5 \%-88.80 \%$ ) for balloon tamponade. It was concluded that balloon tamponade was the least invasive and most rapid remedy for PPH management and should be considered as the first step following failure of medical therapy.

Condom catheter is a cheap and easily available alternative to catheters like Sengstaken Blakemore tube and Bakri balloon which are commonly used for tamponade in post-partum haemorrhage. This prospective study was planned to evaluate the utility of condom catheter tamponade in non-traumatic PPH after failure of medical management. 


\section{METHODS}

Our study was a prospective interventional study conducted at a tertiary care multi-speciality government hospital of New Delhi, India from May 2013 to June 2014. All patients who delivered either vaginally or by Caesarean section and developed non-traumatic postpartum haemorrhage not responding to medical management were included in this study.

The procedure was performed without additional anaesthesia. First, bleeding due to local trauma or retained tissue in the uterus was excluded. Blood loss was estimated by visual method including use of calibrated mops and suction apparatus. A condom was tied over the distal end of the Foley's catheter with a silk thread. Condom-catheter assembly was inserted into the uterine cavity with a sponge holding forceps after introduction of sim's speculum into the vagina and holding cervical lips with sponge holding forceps. Condom was inflated with appropriate volume of normal saline $(250$ to $500 \mathrm{ml})$ till bleeding was arrested and balloon was visible through the cervical canal. Outer end of Foley's catheter was clamped and tied with silk thread. Bleeding was observed for next 15minutes. If bleeding stopped, tamponade was continued for next 12-24hrs. Oxytocin infusion was continued until 12 to 24 hours in the postoperative period. After 12-24 hours, if the uterine fundus remained at the same level and no bleeding was seen through the cervix, balloon was removed. Oxytocin infusion $(20 \mathrm{U}$ in $500 \mathrm{ml}$ Normal Saline) was started half an hour before deflation, if not already on flow. The balloon was deflated slowly (at the rate of 15 to $20 \mathrm{ml}$ per minute) but not removed until next 30 minutes, in case need for re-inflation arose. Oxytocin infusion was continued during deflation. If the patient did not bleed for next 30 minutes, the catheter balloon assembly was removed and oxytocin infusion continued according to clinical assessment, for a maximum of 6 hours.

Statistical analysis was done using simple measures like mean, median etc.

\section{RESULTS}

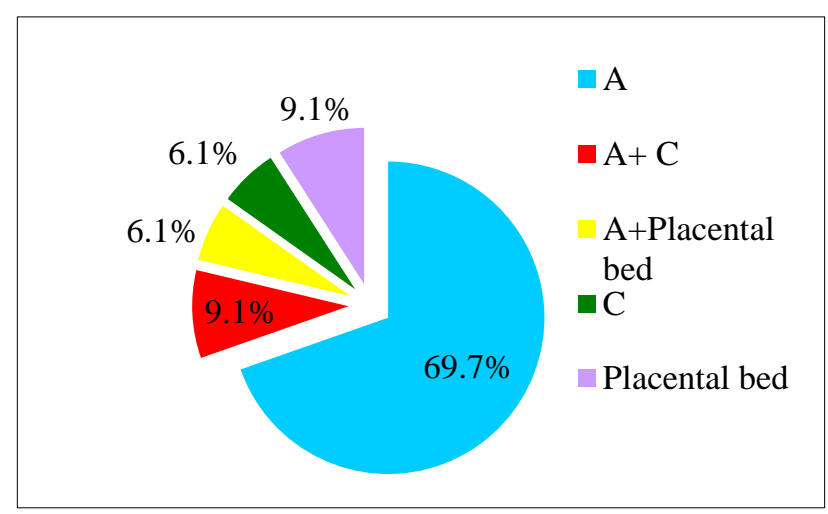

Figure 1: Causes of PPH.
All women included in the study had primary $\mathrm{PPH}$. Atonicity (A) of the uterus was the cause in $23(69.7 \%)$, bleeding placental bed in $3(9.1 \%)$, atonicity with coagulopathy in $3(9.1 \%)$, atonicity with bleeding placental bed in $2(6.1 \%)$ and only coagulopathy(C) in $2(6.1 \%)$ (Figure 1).

In 31 patients $(93.9 \%)$, condom catheter balloon successfully controlled bleeding and no additional intervention was required at the time of deflation. The mean time taken to arrest bleeding after the application of the condom catheter balloon tamponade was 6.66 minutes $[$ Median $(\mathrm{IQR})=6(5-8) ; 95 \% \mathrm{CI}=5.871-7.268$ ] $($ Table 1).

The mean volume of fluid used to create tamponade was $381.06 \mathrm{ml}[$ Median $(\mathrm{IQR})=400(300-450) ; 95 \% \mathrm{CI}=$ 352.275 - 409.845] (Table 2).

On an average the condom catheter tamponade was placed in situ for 19.94 hours [Median (IQR) = $21(16-$ 24); $95 \% \mathrm{CI}=18.578-21.302$ ] (Figure 2).

Table 1: The mean time taken to arrest bleeding after the application of the condom catheter balloon tamponade.

\begin{tabular}{|lll|}
\hline Time taken & Frequency & Percentage \\
\hline NA & 2 & $6.1 \%$ \\
\hline $1-5 \mathrm{~min}$ & 8 & $24.2 \%$ \\
\hline $6-10 \mathrm{~min}$ & 21 & $63.6 \%$ \\
\hline$>10 \mathrm{~min}$ & 2 & $3.0 \%$ \\
\hline Total & 33 & $100 \%$ \\
\hline Mean \pm SD & $6.66 \pm 1.98$ \\
\hline Median (IQR) & $6(5-8)$ \\
\hline $95 \%$ CI & $5.871-7.268$ \\
\hline
\end{tabular}

Table 2: The mean volume of fluid used to create tamponade.

\begin{tabular}{|lll|}
\hline Volume of fluid & Frequency & Percentage \\
\hline$<300 \mathrm{ml}$ & 5 & $15.2 \%$ \\
\hline $300-400 \mathrm{ml}$ & 16 & $48.5 \%$ \\
\hline $400-500 \mathrm{ml}$ & 12 & $36.4 \%$ \\
\hline Total & 33 & $100 \%$ \\
\hline Mean \pm SD & $381.06 \pm 81.18$ \\
\hline Median (IQR) & $400(300-450)$ \\
\hline $95 \%$ CI & $352.275-409.845$ \\
\hline
\end{tabular}

Every patient received 10 to 20 units of oxytocin infusion. Thirty one $(93.9 \%)$ patients received intramuscular methylergometrine $(0.2 \mathrm{mg})$ injection in the range of 0 to 3 doses (average 2.45). Two (6.1\%) women who didn't receive methylergometrine had contraindication for the same. All but one patient received $\mathrm{PGF}_{2}$ alpha (250 microgram) injection in the range of 0 to 5 doses (average of 3.21). All but one woman received $\mathrm{PGE}_{1} 1000$ micrograms (five 200 microgram tablets) per rectum. 


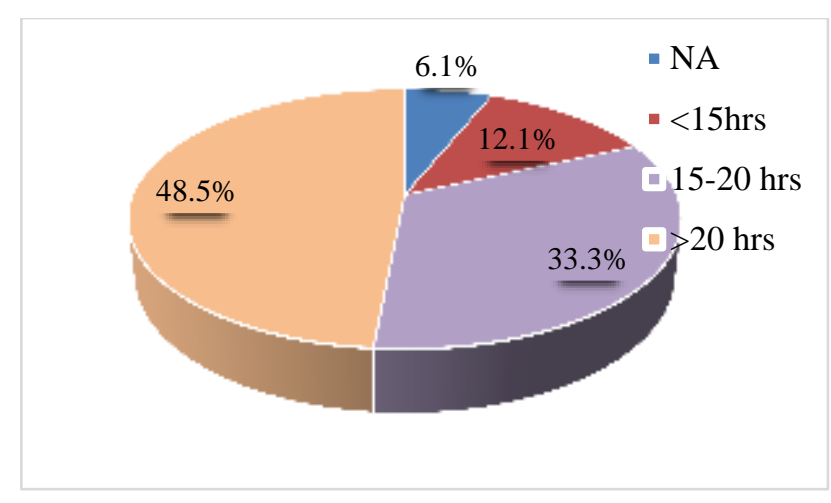

Figure 2: Duration of tamponade.

Average blood loss per patient due to PPH in the study was 1.48 litres (SD 0.46; 95\% CI 1.314-1.643). All 33 patients were transfused with blood or blood products or both. Average whole blood/packed cell transfusions received by the patients in the study was $3.09 \pm 1.355$ units (range $1-7$, total 102 units), 1.73 units of FFP (range 0 to 14; total 57 units) and 1.09 units of platelets ( range 0 to8; total 36 units). The mean duration of hospital stay was 8.88 days. Twenty nine women $(87.9 \%)$ were followed up at 6 weeks postpartum. None of them had any clinical symptoms or signs of endometritis.

In two patients in whom condom catheter balloon tamponade failed to attain haemostasis, hysterectomy was done. One of these two patients had multiple high risk factors including anaemia, antepartum eclampsia, deranged coagulation profile and was shifted to surgical intensive care unit where she expired due to sepsis and multi-organ dysfunction.

\section{DISCUSSION}

In the study period, 12460 deliveries took place of which 184 patients developed non-traumatic PPH. Thirty three patients were enrolled for the study and 23 of those patients (69.7\%) had atonicity as the only cause for PPH while the other ten had a combination of factors such as coagulopathy and bleeding from the placental bed along with atonic uterus. Twenty three $(69.7 \%)$ patients had pre-existing risk factors for PPH such as jaundice, anaemia, preeclampsia etc.

As shown in our study, in 31 out of 33 patients (93.9\%), condom catheter balloon successfully controlled bleeding and no additional intervention was required [95\% CI $79.4-$ 99.3]. Tindell $\mathrm{K}$ et al conducted a systemic analysis to evaluate the effectiveness of various types of uterine balloon tamponade in the management of PPH in resource poor settings. ${ }^{4}$ The studies used various types of uterine balloon tamponade, including condom catheter (number of women $=193)$, Foley's catheter $(\mathrm{n}=5)$, and Sengstaken-Blakemore oesophageal catheter $(\mathrm{n}=1)$. In these studies, uterine balloon tamponade successfully treated intractable PPH in 234 out of 241 women. Akhter $\mathrm{S}$ et al evaluated the efficacy of hydrostatic condom catheter to control PPH due to atonicity and morbid placental adhesions in 23 patients. ${ }^{5}$ The condom was inflated with 250 to $500 \mathrm{ml}$ of normal saline and was kept in situ for 24 to 48 hours. In all 23 cases bleeding was successfully controlled. No patient needed further intervention and no intrauterine infection was documented. Thapa $\mathrm{K}$ et al in their prospective study successfully applied condom catheter tamponade in 14 patients with varying degrees of PPH not controlled with pharmacological measures. ${ }^{6}$ The Foley's - condom catheter tamponade stopped bleeding in all the cases and no complications were encountered. Condous GS et al evaluated the tamponade test in the management of massive PPH in 16 patients and success was met with in $14(87.5 \%)$ of them. ${ }^{7}$ Two $(12.5 \%)$ gave negative tamponade test and hence underwent laparotomy. This diagnostic test rapidly identified patients with $\mathrm{PPH}$ who required a laparotomy. Rathore AM et al in their prospective study conducted from 2009 to 2012 at Maulana Azad Medical College New Delhi India, studied the efficacy of condom catheter tamponade in the management of non-traumatic PPH resistant to medical therapy in 18 patients with a success rate of $94 \%{ }^{8}$ Lydia R et al successfully managed four cases of massive PPH due to uterine atony using condom catheter balloon tamponade. ${ }^{9}$

The mean volume of fluid used to create tamponade and stop bleeding was $381.06 \pm 81.18 \mathrm{ml}$ which was comparable to the studies by Akhtar $\mathrm{S}$ et al (mean reported fluid volume $336.4 \mathrm{ml}$ ) and Rather SY et al (mean reported volume $342.8 \mathrm{ml}$ ). ${ }^{5,10}$ The maximum volume of fluid used in the study was $500 \mathrm{ml}$. Till date the maximum volume of fluid a condom catheter can hold has not been described, so the end point for fluid inflation was till the bleeding stopped and not the upper capacity limit as is the case with other balloon devices like Sengstaken-Blakemore, Foley's catheter Bakri balloon and Rusch urological catheter, all of which have a defined upper limit. Since these devices can hold a limited volume of fluid before they burst, for example $30 \mathrm{ml}$ for Foley's balloon, 500ml for Bakri balloon and $250 \mathrm{ml}$ for the gastric balloon of Sengstaken-Blakemore tube, the tamponade test can be falsely be labelled as negative though these devices can prove useful in cases of small uteri as in post-abortal cases and secondary PPH.

In our study the mean time taken to arrest bleeding after the application of the balloon tamponade was $6.66 \pm 1.98$ minutes which was comparable to the study by Rathore AM et al (6.06 minutes). ${ }^{8}$ While waiting for arrest of haemorrhage, simultaneous arrangements can be done to proceed to surgical measures. So waiting for 10 - 15 minutes for the haemorrhage to stop while patient and operation theatre are prepared for laparotomy can be justified, when weighed against the radical procedures like hysterectomy. Hence guarded wait can prevent radical procedures, more importantly so in primiparous women, and the much valuable uteri can be salvaged.

Average blood loss in this study was 1.48 litres which was comparable to the study by Rathore AM. ${ }^{8}$ Since blood loss estimation was done by visual method methods using 
calibrated mops, it was subject to error. Blood loss estimation by the suction apparatus in cases of caesarean section would have been more objective, but often liquor suction and blood suction were done with the same apparatus which lead to inaccuracies in the blood loss estimation even by this method.

In our study the condom catheter tamponade was placed in situ for a mean duration of $19.94 \pm 3.84$ hours. Minimum duration for which condom catheter balloon tamponade was left in situ was 13 hours and the maximum was 24 hours. In the study by Rathore AM and Thapa $\mathrm{K}$ duration of tamponade was between 24 to 28 hours. ${ }^{6,8}$ The tamponade duration in our study was determined by the factors as the hemodynamic condition of the patient, correction of coagulation abnormality, availability of senior obstetrician, availability of senior anaesthetist. The shorter duration of tamponade in our study can imply that an attempt at earlier deflation of the tamponade can be made, though further studies are needed to recommend a specific duration for the tamponade. Although condom catheter tamponade was deflated at an early interval compared to other studies, haemorrhage didn't resume in any patient. Though no major complications have been reported by any study group with the longer duration of the tamponade, the shorter duration may reduce the morbidity due to longer duration, if any proved in future.

Except for febrile morbidity in five patients in our study, no major short-term complication was noted in any patient. Among these five, cause of febrile morbidity was unknown in one patient only. In the remaining four; respiratory infection in one, urinary tract infection in two and thrombophelebitis at venipuncture site in one patient were identified as the cause. None of the patients had any complaints and no complications like endometritis were noted at six weeks follow up. Long-term complications like development of asherman's syndrome, dysmenorrhoea, oligomenorrhoea or problems with future conception and fertility were not evaluated due to time constraints of the study.

Few technical difficulties that were encountered in our study included expulsion, in one patient who was asthmatic, and abnormally tight tie of the condom over the Foley's catheter leading to inflation failure in one patient.

In the setting of failure of medical management and when other options are not available, especially in low-resource settings, condom catheter balloon is a potential and promising remedy available. It is simple, cheap, easily available, effective and even a trained midwife can apply it. It fits uterine contours naturally and no special technique as in case of uterine packing is needed. It can be used in cases of atonic uterus as well as PPH due to other causes although studies involving larger groups of patients are needed to further investigate this use. Further result in terms of arrest of haemorrhage is available immediately. Even if it fails, application of condom tamponade can buy us time to prepare the patient for other surgical procedures. . In resource-poor countries such as ours, where a majority of deliveries take place at peripheral health centres, there is a continuing need to develop low cost methods requiring minimal skill to stem the morbidity and mortality arising out of postpartum complications.

Limitations of the study include small sample size. Also the impact of condom catheter tamponade on long term outcomes like development of Asherman's syndrome, menstrual disturbances, development of pelvic inflammatory diseases in future, future fertility were not studied due to short duration of the study.

\section{CONCLUSION}

Uterine tamponade using condom catheter balloon to manage non-traumatic PPH is a fairly good procedure with a definite advantage over other tamponade procedures with respect to the cost, the expertise, and availability. It also has the admirable advantage of preservation of uterus and hence the future menstrual function, fertility and conception when compared to the radical procedures like hysterectomy. It is advantageous over other surgical procedures like compression sutures and devascularisation in terms of the cost effectiveness, the ease of the procedure and the duration of hospital stay, provided haemostasis is attained.

\section{Funding: No funding sources}

Conflict of interest: None declared

Ethical approval: The study was approved by the Institutional Ethics Committee

\section{REFERENCES}

1. Abouzahn C. Global burden of maternal death and disability. Br Med Bull. 2003;67:1-11.

2. Karoshi M, Keith L. Challenges in managing postpartum haemorrhage in resource- poor countries. Clin Obstet Gynecol. 2009;52:285-98.

3. Doumouchtsis SK, Papageoeghiou AT, Arulkumaran S. Systematic review of conservative management of postpartum haemorrhage: what to do when medical management fails. Obstet Gynecol Surv 2007;62(8):540-7.

4. Tindell K Garfinkel R, Abu-Hayder E, Ahn R, Burke $\mathrm{T}$, Conn K. Uterine balloon tamponade for the treatment of postpartum haemorrhage in resourcepoor settings: a systematic review. $\mathrm{Br} \mathrm{J}$ Obstet Gynecol. 2012;120(1):5-14.

5. Akhter S, Begum MR, Kabir Z, Rashid M, Laila TR, Zabeen F. Use of a condom to control massive postpartum haemorrhage. Med Gen Med. 2003;5(3):38.

6. Thapa K, Malla B, Pandey S, Amatya S. Intrauterine condom tamponade in management of postpartum haemorrhage. J Nepal Health Res Counc. 2010;8:19.

7. Condous GS, Arulkumaran S, Symonds I, Chapman $\mathrm{R}$, Sinha A, Razvi K. The 'tamponade test' in the 
management of massive postpartum haemorrhage. Obstet Gynecol. 2003;101:767-72.

8. Rathore AM, Gupta S, Manaktala U, Gupta S, Dubhey C, Khan AM. Uterine tamponade using condom catheter balloon in the management of nontraumatic postpartum haemorrhage. J Obstet Gynaecol Res. 2012;38:1162.
9. Airede LR, Nnadi DC. The use of condom catheter for the treatment of postpartum haemorrhage. Trop Doct. 2008;38:84-6.

10. Rather SY, Qadir A, Parveen S, Jabeen F. Use of condom to control intractable PPH. JK Science. 2010;12(3):127-9.

Cite this article as: Ahmad SN, Seth S, Agarwal S, Choudhary S. Evaluating condom catheter balloon tamponade in non-traumatic postpartum haemorrhage resistant to medical management. Int J Reprod Contracept Obstet Gynecol 2016;5:1874-8. 